\title{
Pfannenstiel incision for surgical excision of a huge pelvi-abdominal cystadenoma: a case report
}

\author{
Ibrahim Abdelazim ${ }^{1,2}$, Mohannad AbuFaza ${ }^{1}$ \\ ${ }^{1}$ Ahmadi Hospital, Kuwait Oil Company, Ahmadi, Kuwait \\ ${ }^{2}$ Ain Shams University, Cairo, Egypt
}

\begin{abstract}
A 56-year-old post-menopausal woman, with 3 previous caesarean sections (CSs), presented to the emergency department with abdominal distension, without abdominal pain, tenderness, and/or rigidity.

The abdominal examination of the studied woman showed a mobile, pelvi-abdominal mass 4 fingers breadth above the umbilicus.

Magnetic resonance imaging study of the mass showed a large, well-defined, multi-locular cystic mass measuring $25.5 \times 21 \mathrm{~cm}$, which was most probably a right ovarian cystadenoma. The studied woman signed a written consent form for total abdominal hysterectomy and bilateral salpingo-oophorectomy, after the normal tumour markers, and pre-operative investigations.

Under general anaesthesia, an elliptical Pfannenstiel skin incision was done to remove the old CSs scars, followed by opening of the patient's anterior abdominal wall in layers. Total abdominal hysterectomy and unilateral left SO were done first, to deliver the ovarian mass easily and intact outside the abdomen after the uterus.

Due to failure to deliver the mass outside the abdomen after removal of the uterus, the right infundibulopelvic ligament was ligated behind the mass, while the mass was still inside the abdomen.

A longitudinal midline incision in the upper flap of the rectus sheath (not involving the skin) was added to deliver the excised right ovarian mass outside the abdomen.

Successfully, the right ovarian mass delivered intact outside the abdomen after the added longitudinal midline incision. This report highlights that the midline vertical incision is not the standard abdominal incision. Moreover, the transverse Pfannenstiel incision is cosmetically better, and should be routinely used to avoid unnecessary vertical abdominal incision.
\end{abstract}

Key words: Pfannenstiel, incision, huge, cystadenoma.

\section{Introduction}

The incidence of benign ovarian cysts in postmenopausal women is about 14-18\% [1]. Mucinous cystadenoma is an ovarian surface epithelium tumour $[2,3]$. It constitutes $15 \%$ of all ovarian tumours [3, 4]. Mucinous cystadenoma usually appears during reproductive age as a large multilocular cyst with smooth capsule $[3,4]$. Ovarian mucinous tumours are benign in approximately $80 \%$ of cases, and rarely appear after the menopause $[3,4]$.

The presence of large mucinous cystadenoma in the ovary $(>5 \mathrm{~cm})$ increases risk of ovarian torsion [4]. The preoperative differentiation between benign and malignant ovarian masses (OMs) is crucial to decide further management [5-7].

Cancer antigen-125 (CA-125) is a tumour marker that could exclude malignancy or endometrioma in suspected OMs $[4,8]$.
Ultrasound is the first imaging tool in cases of suspected OMs [4, 8]. Magnetic resonance imaging is helpful in demonstrating the internal components of the suspected OMs [9]. Despite the recent advances in imaging tools, the early diagnosis of ovarian cancers is still difficult [4].

Chen et al. found that the IOTA-ADNEX model had good to excellent performance in distinguishing between benign and malignant OMs in a Chinese setting [10].

Nowak et al. also concluded that both the IOTA and CA-125 are complementary and can be used to differentiate between malignant and benign OMs [11].

This report highlights that the midline vertical incision is not the standard abdominal incision. Moreover, the transverse Pfannenstiel incision is cosmetically better and should be routinely used to avoid unnecessary vertical abdominal incision. 


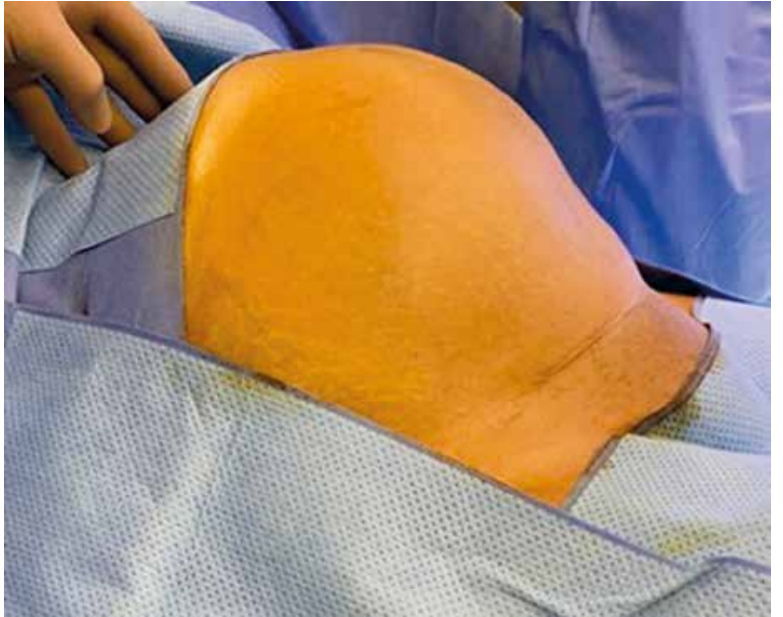

Fig. 1. The studied pelvi-abdominal mass reaching 4 fingers breadth above the umbilicus

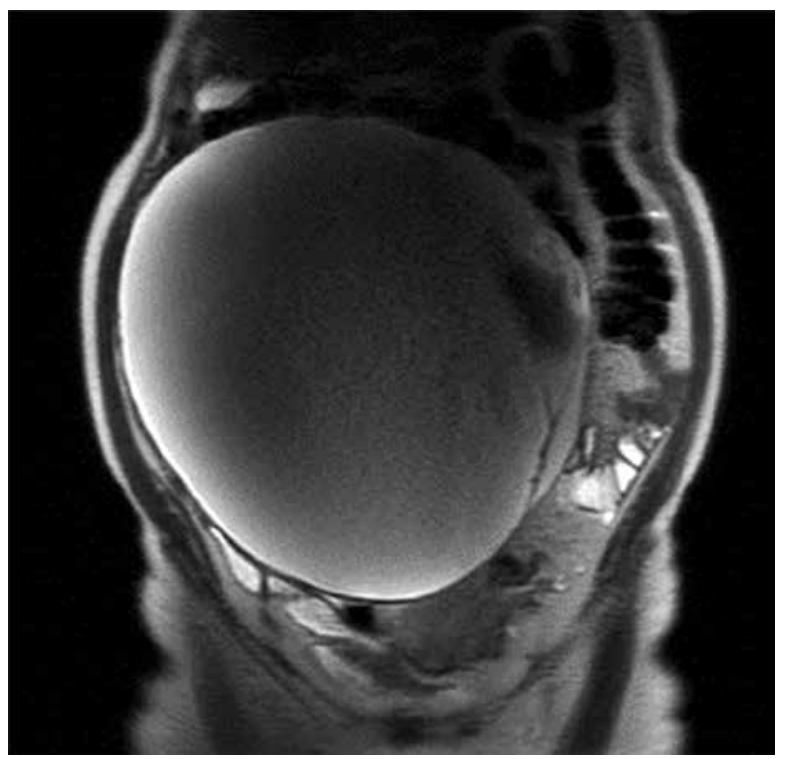

Fig. 2. Magnetic resonance imaging of the studied pelvi-abdominal mass $(25.5 \times 21 \mathrm{~cm})$

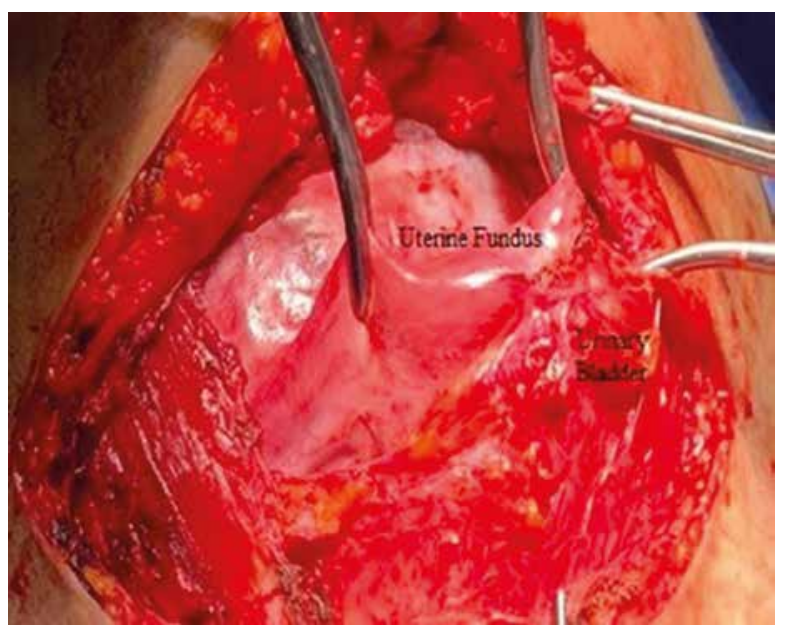

Fig. 3. The urinary bladder of the studied woman was firmly adherent to the anterior uterine wall up to its fundus

\section{Case report}

A 56-year-old post-menopausal woman, menopausal over the last 7 years, with 3 previous caesarean sections (CSs), hypertensive on antihypertensive medications presented to the emergency department with abdominal distension, without abdominal pain, tenderness, and/or rigidity.

She was vitally stable (blood pressure $110 / 70 \mathrm{mmHg}$, pulse rate $88 /$ mins.), her body weight was $68 \mathrm{~kg}$, and body mass index was $27.9 \mathrm{~kg} / \mathrm{m}^{2}$.

The abdominal examination of the studied woman showed mobile, pelvi-abdominal mass 4 fingers breadth above the umbilicus ( 32 weeks` gestation size) for imaging evaluation, and tumour markers (Fig. 1)

Magnetic resonance imaging study of the mass with intravenous contrast showed a large, well-defined T1-hypo and T2-hyper-intense multi-locular right cystic mass measuring $25.5 \times 21 \mathrm{~cm}$, arising from the pelvis to the upper abdomen with a well-defined capsule. There was no evidence of solid and/or mural nodules within the cyst. The lesion caused displacement of the surrounding structures, reaching the abdominal wall anteriorly and the anterior vertebral margin posteriorly (Fig. 2).

It was most probably a right ovarian cystadenoma. The pre-operative tumour markers, including CA-125 31.9 (normal 0-35 $\mathrm{mlU} / \mathrm{mL}$ ), carcinoembryonic antigen 1.71 (normal 4.7-5.2 ng/mL), $\beta$-hCG 4.4 (normal 0-10 $\mathrm{mIU} / \mathrm{mL}$ ), and $\alpha$ fetoprotein 2.72 (normal $0-6 \mathrm{IU} / \mathrm{mL}$ ), were normal.

The studied woman signed a written consent form for total abdominal hysterectomy and bilateral salpingo-oophorectomy, after the normal tumour markers, and preoperative investigations.

Under general anaesthesia, an elliptical Pfannenstiel skin incision was done to remove the old CSs scars, followed by opening of patient's anterior abdominal wall in layers.

The urinary bladder was firmly adherent to anterior uterine wall up to its fundus (Fig. 3), and the OMs originated from the right ovary with a thick capsule.

The urinary bladder was dissected successfully from the anterior uterine wall to the cervix. Total abdominal hysterectomy and unilateral left SO was done first, to deliver the OMs easily and intact outside the abdomen after the uterus.

Due to failure to deliver the OMs outside the abdomen after removal of the uterus through the Pfannenstiel incision, the right infundibulopelvic ligament was ligated behind the mass, while the mass was still inside the abdomen. A longitudinal midline incision in the upper flap of the rectus sheath (not involving the skin) was added to deliver the excised right OM outside the abdomen. Successfully, the right OM was delivered intact outside the abdomen after the added longitudinal midline incision. 
During closure of the anterior abdominal wall in layers, the length of the Pfannenstiel incision and the added longitudinal incision were measured (18 and $5 \mathrm{~cm}$, respectively) (Fig. 4).

The size of excised OMs was $25.5 \times 21 \mathrm{~cm}$ (Fig. 5), and its weight was $4.505 \mathrm{~kg}( \pm 10 \%)$ (Fig. 6).

The histological examination of the excised $\mathrm{OM}$ showed a multilocular cyst lined by flat columnar and cuboidal epithelium with underlying fibrous connective stroma of spindle cells, confirming the preoperative provisional diagnosis of ovarian mucinous cystadenoma. The studied woman was discharged from the hospital on the $5^{\text {th }}$ post-operative day (PO) in good genal condition. Written consent was obtained from the studied woman to publish her data and images as a case report.

\section{Discussion}

Transverse abdominal incision should be recommended because it has fewer early PO complications (pain, burst abdomen, and pulmonary morbidity), and lower incidence of late incisional hernias compared to vertical abdominal incisions.

In addition, transverse incision offers good access to intra-abdominal structures as well as the vertical incision [12]. A vertical midline incision is still the incision of choice in conditions that require rapid intra-abdominal entry (such as trauma) or when the preoperative diagnosis is uncertain [12].

A systematic review by Grantcharov et al. found that the transverse abdominal incisions had less PO pain and fewer pulmonary complications, and the odds of burst abdomen and late incisional hernia after vertical abdominal incisions were 2.86 and 1.68 , respectively [12].

Seiler et al. found that both the vertical and transverse abdominal incisions were similar with no difference regarding PO pain, pulmonary complications, PO hospital stay, and incisional hernias [13].

Seiler et al. concluded that the decision to use an abdominal incision should depend on the surgeon's preference, respecting the patient's disease and anatomy [13].

There is wrong believe that the midline vertical incision is the only standard abdominal incision. Moreover, the basic rule in surgery necessitates a comfortable incision for the surgeon, which provides adequate access to the pathology. The transverse Pfannenstiel incision is a popular incision in pelvic surgery; it is cosmetically better and should be routinely used to avoid unnecessary vertical abdominal incision [14].

This report represents a huge pelvi-abdominal cystadenoma $(25.5 \times 21 \mathrm{~cm})$ surgically excised through Pfannenstiel, and a small added longitudinal midline incision, to highlight that the midline vertical incision is not the standard abdominal incision.
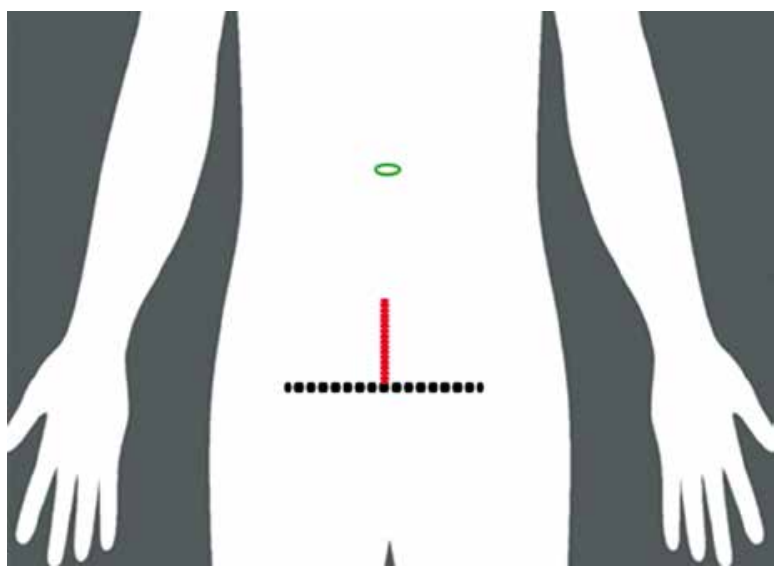

Fig. 4. The length of the Pfannenstiel incision (black line) and the added midline longitudinal incision (red line) in the upper flap of the rectus sheath to deliver the excised mass outside the abdomen ( $18 \times 5 \mathrm{~cm}$, respectively)

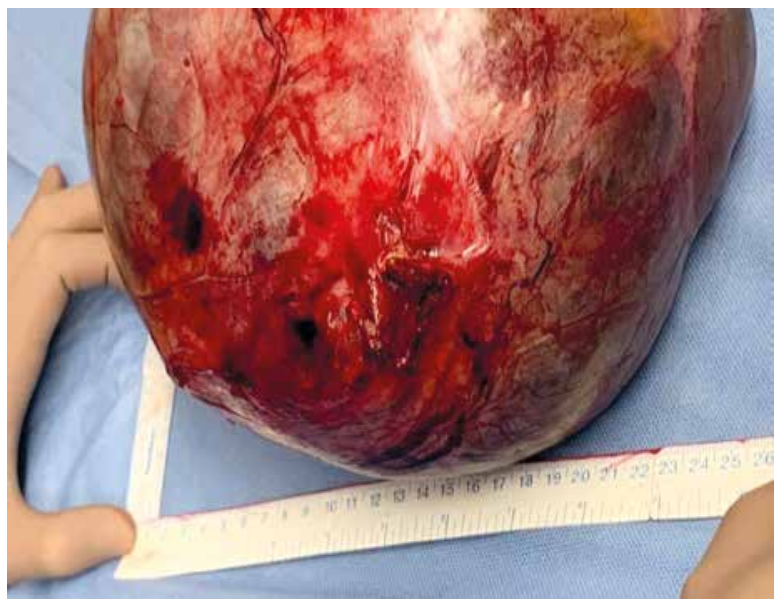

Fig. 5. The size of the surgical excised ovarian cystadenoma $(25.5 \times 21 \mathrm{~cm})$

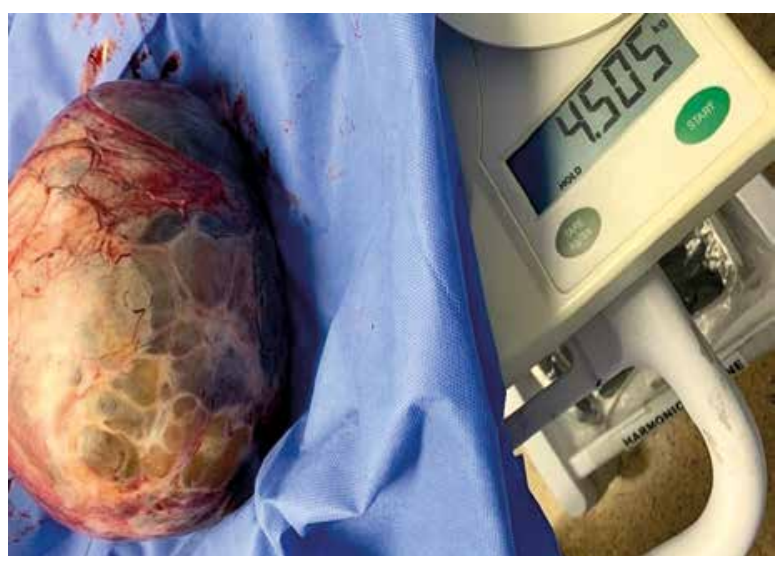

Fig. 6. The weight of the surgically excised ovarian cystadenoma $(4.505 \mathrm{~kg} \pm 10 \%)$

\section{Conclusions}

This report highlights that the midline vertical incision is not the standard abdominal incision. Moreover, the transverse Pfannenstiel incision is cosmetically bet- 
ter, and it should be routinely used to avoid unnecessary vertical abdominal incision.

\section{Acknowledgement}

The authors are grateful to the studied woman who agreed and gave consent to publish her data and images as a case report.

Written consent was obtained from the studied woman to publish her data and images as a case report.

\section{Disclosure}

The authors report no conflict of interest.

\section{References}

1. Mimoun C, Fritel X, Fauconnier A, Deffieux X, Dumont A, Huchon C. [Epidemiology of presumed benign ovarian tumors]. J Gynecol Obstet Biol Reprod (Paris) 2013; 42: 722-729.

2. Marret H, Cayrol M. [Sonographic diagnosis of presumed benign ovarian tumors]. J Gynecol Obstet Biol Reprod (Paris) 2013; 42: 730-743.

3. Kamel RM. A massive ovarian mucinous cystadenoma: a case report. Reprod Biol Endocrinol 2010; 8: 24

4. Abdelazim IA, AbuFaza M, Bekmukhambetov Y, Zhurabekova G, Shikanova S. Postmenopausal adnexal torsion: rare case report. Menopause Rev 2020; 19: 49-51.

5. Javdekar R, Maitra N. Risk of malignancy index (RMI) in evaluation of adnexal mass. J Obstet Gynaecol India 2015; 65: 117-121.

6. Sönmezer M, Taşkın S. Fertility preservation in women with ovarian endometriosis. Womens Health (Lond) 2015; 11: 625-631.

7. Adilgereyeva AS, Abdelazim IA, Zhurabekova GA. Clinical and pathological features of women with adnexal masses admitted as emergency cases to the Gynaecology Department of West Kazakhstan University. Menopause Rev 2019; 18: 180-183.

8. Huang C, Hong MK, Ding DC. A review of ovary torsion. Ci Ji Yi Xue Za Zhi 2017; 29: 143-147.

9. Naffaa L, Deshmukh T, Tumu S, Johnson C, Boyd KP, Meyers AB. Imaging of acute pelvic pain in girls: ovarian torsion and beyond. Curr Probl Diagn Radiol 2017; 46: 317-329.

10. Chen H, Qian L, Jiang M, Du Q, Yuan F, Feng W. Performance of IOTAADNEX model in evaluating adnexal masses in a gynecological oncology center in China. Ultrasound Obstet Gynecol 2019; 54: 815-822.

11. Nowak A, Soja M, Masternak M, Mokros Ł, Wilczyński J, Szubert M. Evaluation of adnexal tumours in the International ovarian tumor analysis system in reference to histopathological results. Menopause Rev 2019; 18: 141-145.

12 Grantcharov TP, Rosenberg J. Vertical compared with transverse incisions in abdominal surgery. Eur J Surg 2001; 167: 260-267.

13. Seiler CM, Deckert A, Diener MK, et al. Midline versus transverse incision in major abdominal surgery: a randomized, double-blind equivalence trial (POVATI: ISRCTN60734227). Ann Surg 2009; 249: 913-920.

14 Abdominal incisions in general surgery: a review. Ann lb Postgrad Med 2007; 5: 59-63. 